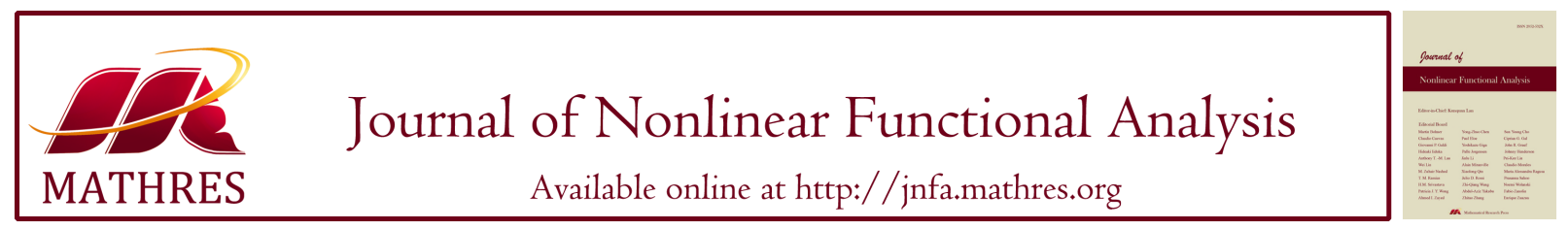

\title{
DYNAMICS OF A STOCHASTIC SHEEP BRUCELLOSIS MODEL WITH IMMIGRATION
}

\author{
LEI DANG, XAMXINUR ABDURAHMAN*
}

College of Mathematics and System Sciences, Xingjiang University, Urumqi 830046, China

\begin{abstract}
Sheep brucellosis is one of the principal infective and contagious bacterial diseases among sheep populations in pastoral areas of China. In this paper, we establish a stochastic sheep brucellosis model by involving the effect of environmental white noise for the propagation process of brucellosis. We use the stochastic Lyapunov function theory to construct a suitable stochastic Lyapunov function for the existence of global positive solution. We also obtain the sufficient conditions for the disease extinction and the disease persistence.
\end{abstract}

Keywords. Stochastic sheep brucellosis model; Threshold value; Extinction; Persistence in the mean.

\section{INTRODUCTION}

Brucellosis, which is also known as the undulant fever, the Mediterranean fever or the Malta fever, is a zoonosis, and the infection is almost invariably transmitted by direct or indirect contact with infected animals or their products [1]. The disease is caused by the bacteria of the genus brucella. There are six species: Brucella abortus, Brucella melinitis, Brucella suis, Brucella ovis, Brucella canis and Brucella neotomae [2]. British military doctor Bruce was the first to confirm the pathogen of the disease in 1886. Hence the disease was named "brucellosis" in order to honor him [3,4]. Brucellosis mostly infects sheep and cattle. It also contaminates dogs, horses, elks, swine, and humans. Infections in sheep is highly contagious because of the pathogenicity of Brucella melitensis and the infected sheep exhibit symptoms which may include abortion during the last third of pregnancy, retained afterbirth, and week calves at birth. The brucellosis organism is shed into the environment by the millions in the afterbirth and fluids associated with calving and aborting, so brucella also widely exists in the environment near or in the farms and it leads to indirect transmission about brucella-to-sheep for brucellosis [7]. As a result of brucellosis, infection can cause sheep abortion. It leads serious economic

${ }^{*}$ Corresponding author.

E-mail addresses: 2956373863@qq.com (L. Dang), xamxinur@sina.com (X. Abdurahman).

Received December 24, 2019; Accepted April 30, 2020.

(C)2020 Journal of Nonlinear Functional Analysis 
consequence for the Chinese sheep industry. Control measures for brucellosis with livestock include quarantine, livestock vaccination and the elimination of infected animals [5].

Mathematical models have been extensively used in researching into not only the transmission dynamics but also the epidemiological characteristics of brucellosis [6]; see, for example, [7, 8, 9, 10] and the references cited therein. In particular, Sun and Zhang [7] studied the following sheep brucellosis model with immigration

$$
\left\{\begin{array}{l}
\frac{\mathrm{d} S}{\mathrm{~d} t}=A+b(S+V)+\delta V-(d+r) S-\beta S(E+I)-\beta_{1} S W \\
\frac{\mathrm{d} E}{\mathrm{~d} t}=\beta S(E+I)+\beta_{1} S W-(d+m) E, \\
\frac{\mathrm{d} I}{\mathrm{~d} t}=m E-(d+\alpha) I, \\
\frac{\mathrm{d} V}{\mathrm{~d} t}=r S-(\delta+d) V \\
\frac{\mathrm{d} W}{\mathrm{~d} t}=k(E+I)-(n \tau+\varepsilon) W,
\end{array}\right.
$$

where $S(t), E(t), I(t)$ and $V(t)$ represent the number of the susceptible, the exposed (in this compartment, they are asymptomatic infectious sheep), the infected and the vaccinated for sheep at time $t$, respectively. According to [11], we assume the average number of brucella that is enough for a sheep to be an infected as an infectious unit. Here $W(t)$ denotes the number of infectious unit in the environment. The spread of brucellosis among sheep is through the infection of the respiratory tract, oral mucosa, mucous membranes and skin. On the other hand, exposed and infected sheep can shed brucella into the environment due to abortion or sheep secretions, which can survive for several weeks, or even months in the feces or contaminated environment under suitable conditions [11]. Therefore, an infected or exposed sheep infects others by two ways: the direct and indirect forms of transmission. $A$ is the immigration rate of $S(t), b$ is the birth rate of the compartment $S(t)$ and $V(t), \delta$ is the losing immunity rate of $V(t), d$ is the output occurs rate of $S(t), \beta$ is the transmission rate coefficient between compartment $S(t)$ and $I(t), \beta_{1}$ is the transmission rate coefficient between compartment $S(t)$ and $W(t), m$ is the transfer rate of sheep from compartment $E(t)$ to compartment $I(t)$, and $\alpha$ is the disease-related death rate of $I(t)$. In $W(t)$, the brucella shedding rate from $E(t)$ and $I(t)$ is $k, \varepsilon$ is the decaying rate of brucella, the sterilization number of the environment is $n$ and the effective sterilization rate at a time is $\tau$, respectively. All parameter values are usually assumed to be nonnegative.

However, in the real world, epidemic systems are inevitably subjected to environmental white noise. We know that environmental perturbations have significant effects on the development and transmission of an epidemic disease. Moreover, in virtue of continuous fluctuations in the environment, the parameters introduced in the system often fluctuate around some average values. Hence, a stochastic epidemic model has more practical significance. There are various possible methods for applying the modeling skills of stochastic differential equations to introduce environmental variability into epidemic models $[6,12,13,14,15,16,17,18,19,20,21]$. Particularly, Zhao and Jiang [18] investigated the dynamics of a stochastic SIRS epidemic model with saturated incidence. They obtained a threshold of the stochastic system, which determines the extinction and persistence of epidemic disease. In [19], Liu and Jiang concerned with the dynamical behavior of a stochastic SIQR epidemic model with standard incidence, which is disturbed by both white and telegraph noises. They obtained sufficient conditions for the existence of positive recurrence of the solutions. They also obtained the sufficient conditions for extinction and persistence in the mean of the disease. Li, Meng and Wang [20] proposed a high-dimensional stochastic SEIQI epidemic model with quarantine-adjusts incidence and the 
imperfect vaccination. The sufficient conditions on the extinction almost surely of the disease and the existence of stationary distribution of the model are established. Chen, Wen and Teng [21] discussed the global dynamics for a stochastic SIS epidemic model with isolation. They obtained the threshold of the system for the extinction and permanence in the mean of the disease. They also discussed the existence of a unique stationary distribution and established the sufficient conditions by using the Lyapunov function method. In [6], Wang et al. discussed nontrivial periodic solutions for a stochastic brucellosis model. They established the existence of nontrivial positive periodic solutions by Has'minskill theory of periodic solutions and constructed a novel combination of Lyapunov functions.

In this paper, to make system (1.1) more reasonable and realistic, we assume that stochastic perturbations are of the white noise type, which are directly proportional to $S(t), E(t), I(t)$, $V(t)$, and $W(t)$, influenced on the $\frac{\mathrm{d} S}{\mathrm{~d} t}, \frac{\mathrm{d} E}{\mathrm{~d} t}, \frac{\mathrm{d} I}{\mathrm{~d} t}, \frac{\mathrm{d} V}{\mathrm{~d} t}$, and $\frac{\mathrm{d} W}{\mathrm{~d} t}$ in system (1.1). Then, corresponding to system (1.1), a stochastic version can be reached by

$$
\left\{\begin{array}{l}
\mathrm{d} S=\left[A+b(S+V)+\delta V-(d+r) S-\beta S(E+I)-\beta_{1} S W\right] \mathrm{d} t+\sigma_{1} S \mathrm{~d} B_{1}(t), \\
\mathrm{d} E=\left[\beta S(E+I)+\beta_{1} S W-(d+m) E\right] \mathrm{d} t+\sigma_{2} E \mathrm{~d} B_{2}(t), \\
\mathrm{d} I=[m E-(d+\alpha) I] \mathrm{d} t+\sigma_{3} I \mathrm{~d} B_{3}(t), \\
\mathrm{d} V=[r S-(\delta+d) V] \mathrm{d} t+\sigma_{4} V \mathrm{~d} B_{4}(t), \\
\mathrm{d} W=[k(E+I)-(n \tau+\varepsilon) W] \mathrm{d} t+\sigma_{5} W \mathrm{~d} B_{5}(t),
\end{array}\right.
$$

where $B_{i}(t)(i=1,2,3,4,5)$ are independent standard Brownian motion defined on a complete probability space $(\Omega, \mathscr{F}, \mathrm{P})$ with a filtration $\left\{\mathscr{F}_{t}\right\}_{t \geq 0}$ satisfying the usual conditions (i.e., it is increasing and right continuous while $\mathscr{F}_{0}$ contains all P-null sets $), \sigma_{i}(t)(i=1,2,3,4,5)$ are continuous and bounded functions for any $t \geq 0$ and $\sigma_{i}^{2}(t)(i=1,2,3,4,5)$ are the intensity of the white noise.

Our purpose is to analyze the existence and uniqueness of global positive solution for model (1.2), the extinction and persistence of the disease. We establish two sufficient conditions to guarantee the extinction and persistence in the mean of the disease with probability one by using the theory of stochastic processes, the Itô's formula and the Lyapunov function method. This paper is organized as follows. In Section 2, we introduce the preliminaries and some lemmas. In Section 3, we show that there is a unique global positive solution of system (1.1). In Section 4, we establish sufficient condition for the extinction of the disease. In Section 5, the criteria on the persistence in the mean of the disease with probability one are stated and proved. In Section 6, some numerical simulations are given to support our theory results. In 7, the last section, we have some necessary discussions.

\section{PRELIMINARIES}

For simplicity and convenience, we introduce the following notations:

(1) $\mathbb{R}_{+}^{5}=\left\{\left(x_{1}, x_{2}, x_{3}, x_{4}, x_{5}\right) \in \mathbb{R}^{5}: x_{i}>0, i=1,2,3,4,5\right\}$.

(2) For an integrable function $f(t) \in[0,+\infty),\langle f(t)\rangle=\frac{1}{t} \int_{0}^{t} f(r) \mathrm{d} s$.

(3) $a \vee b=\max \{a, b\}, a \wedge b=\min \{a, b\}$.

In general, we consider the $n$-dimensional stochastic differential equation

$$
\mathrm{d} x(t)=h(x(t), t) \mathrm{d} t+g(x(t), t) \mathrm{d} B(t) \quad \text { for } t \geq t_{0},
$$


with initial value $x\left(t_{0}\right)=x_{0} \in \mathbb{R}^{n}$. Denote by $C^{1,2}\left(\mathbb{R}^{n} \times\left[t_{0}, \infty\right] ; \mathbb{R}_{+}\right)$the set of all nonnegative functions $V(x, t)$ defined on $\mathbb{R}^{n} \times\left[t_{0}, \infty\right]$ such that they are continuously twice differentiable in $x$ and once in $t$. The differential operator $\mathscr{L}$ associated with Equation (2.1) is defined by

$$
\mathscr{L}=\frac{\partial}{\partial t}+\sum_{i=1}^{n} h_{i}(x, t) \frac{\partial}{\partial x_{i}}+\frac{1}{2} \sum_{i, j=1}^{n}\left[g^{T}(x, t) g(x, t)\right]_{i j}\left(\frac{\partial^{2}}{\partial x_{i} \partial x_{j}}\right) .
$$

If $\mathscr{L}$ acts on a function $V \in C^{1,2}\left(\mathbb{R}^{n} \times\left[t_{0}, \infty\right] ; \mathbb{R}_{+}\right)$, then

$$
\mathscr{L} V(x, t)=V_{t}(x, t)+V_{x}(x, t) f(x, t)+\frac{1}{2} \operatorname{trace}\left[g^{T}(x, t) V_{x x} g(x, t)\right],
$$

where $V_{t}=\frac{\partial V}{\partial t}, V_{x}=\left(\frac{\partial V}{\partial x_{1}}, \ldots, \frac{\partial V}{\partial x_{n}}\right)$, and $V_{x x}=\left(\frac{\partial^{2} V}{\partial x_{i} \partial x_{j}}\right)_{n \times n}$. In view of the generalized Itô's formula, if $x(t) \in \mathbb{R}^{n}$, then

$$
\mathrm{d} V(x(t), t)=\mathscr{L} V(x(t), t) \mathrm{d} t+V_{x}(x(t), t) g(x(t), t) \mathrm{d} B(t) .
$$

For other preliminaries, we give the following lemmas.

Lemma 2.1. For deterministic model (1.1), let $\mathscr{R}_{0}=\frac{A(d+\delta)(d+\alpha+m)}{(d-b)(d+\delta+r)(d+\alpha)(d+m)}\left(\beta+\frac{\beta_{1} k}{\varepsilon+n \tau}\right)$. We have following conclusions.

(1) The disease-free equilibrium $P_{0}\left(S^{0}, 0,0, V^{0}, 0\right)$ of system (1.1) is globally asymptotically stable when $\mathscr{R}_{0}<1$, where $S^{0}=\frac{A(\delta+d)}{(d-b)(\delta+d+r)}$, and $V^{0}=\frac{A r}{(d-b)(\delta+d+r)}$.

(2) The unique endemic equilibrium $P^{*}\left(S^{*}, E^{*}, I^{*}, V^{*}, W^{*}\right)$ of system (1.1) is globally asymptotically stable when $\mathscr{R}_{0}>1$, where $S^{*}=\frac{(d+m)(d+\alpha)}{(d+m+\alpha)\left(\beta+\frac{\beta_{1} k}{\varepsilon+n \tau}\right)}, E^{*}=\frac{(d+r+\delta)(d-b)(d+\alpha)\left(\mathscr{R}_{0}-1\right)}{(d+\delta)(d+m+\alpha)\left(\beta+\frac{\beta_{1} k}{\varepsilon+n \tau}\right)}$, $I^{*}=\frac{m E^{*}}{d+\alpha}, V^{*}=\frac{r S^{*}}{(d+\delta)}$, and $W^{*}=\frac{k(\alpha+d+m) E^{*}}{(n \tau+\varepsilon)(d+\alpha)}$.

Lemma 2.2. Let $(S(t), E(t), I(t), V(t), W(t))$ be the solution of model (1.2) with initial value $(S(0), E(0), I(0), V(0), W(0)) \in \mathbb{R}_{+}^{5}$, then

$$
\begin{gathered}
\limsup _{t \rightarrow \infty}(S(t)+E(t)+I(t)+V(t))<\infty \text { a.s. } \\
\limsup _{t \rightarrow \infty} W(t)<\infty \text { a.s. }
\end{gathered}
$$

Moreover,

$$
\begin{gathered}
\limsup _{t \rightarrow \infty}\langle S(t)+E(t)+I(t)+V(t)\rangle \leq \frac{A}{d-b} \text { a.s. } \\
\limsup _{t \rightarrow \infty}\langle W(t)\rangle \leq \frac{k A}{(d-b)(n \tau+\varepsilon)} \text { a.s. }
\end{gathered}
$$

Proof. By using model (1.2), we have

$$
\begin{aligned}
\mathrm{d}(S(t)+E(t)+I(t)+V(t))= & {[A-d(S+E+I+V)+b(S+V)-\alpha I)] \mathrm{d} t+\sigma_{1} S \mathrm{~d} B_{1}(t) } \\
& +\sigma_{2} E \mathrm{~d} B_{2}(t)+\sigma_{3} I \mathrm{~d} B_{3}(t)+\sigma_{4} V \mathrm{~d} B_{4}(t)+\sigma_{5} W \mathrm{~d} B_{5}(t) \\
\leq & {[A-(d-b)(S+E+I+V)-\alpha I)] \mathrm{d} t+\sigma_{1} S \mathrm{~d} B_{1}(t) } \\
& +\sigma_{2} E \mathrm{~d} B_{2}(t)+\sigma_{3} I \mathrm{~d} B_{3}(t)+\sigma_{4} V \mathrm{~d} B_{4}(t)+\sigma_{5} W \mathrm{~d} B_{5}(t) .
\end{aligned}
$$


Making use of the comparison theorem of stochastic differential equation [22], one sees that

$$
\begin{aligned}
& S(t)+E(t)+I(t)+V(t) \\
\leq & \frac{A}{d-b}+\left[S(0)+E(0)+I(0)+V(0)-\frac{A}{d-b}\right] e^{-(d-b) t} \\
& -\alpha \int_{0}^{t} e^{-(t-r)(d-b)} I(r) \mathrm{d}(r)+\sigma_{1} \int_{0}^{t} e^{-(t-r)(d-b)} S(r) \mathrm{d} B_{1}(r) \\
& +\sigma_{2} \int_{0}^{t} e^{-(t-r)(d-b)} E(r) \mathrm{d} B_{2}(r)+\sigma_{3} \int_{0}^{t} e^{-(t-r)(d-b)} I(r) \mathrm{d} B_{3}(r) \\
& +\sigma_{4} \int_{0}^{t} e^{-(t-r)(d-b)} V(r) \mathrm{d} B_{4}(r)+\sigma_{5} \int_{0}^{t} e^{-(t-r)(d-b)} W(r) \mathrm{d} B_{5}(r) \\
\leq & \frac{A}{d-b}+\left[S(0)+E(0)+I(0)+V(0)-\frac{A}{d-b}\right] e^{-(d-b) t}+M(t) a . s .,
\end{aligned}
$$

where

$$
\begin{aligned}
M(t)= & \sigma_{1} \int_{0}^{t} e^{-(t-r)(d-b)} S(r) \mathrm{d} B_{1}(r)+\sigma_{2} \int_{0}^{t} e^{-(t-r)(d-b)} E(r) \mathrm{d} B_{2}(r) \\
& +\sigma_{3} \int_{0}^{t} e^{-(t-r)(d-b)} I(r) \mathrm{d} B_{3}(r)+\sigma_{4} \int_{0}^{t} e^{-(t-r)(d-b)} V(r) \mathrm{d} B_{4}(r) \\
& +\sigma_{5} \int_{0}^{t} e^{-(t-r)(d-b)} W(r) \mathrm{d} B_{5}(r) .
\end{aligned}
$$

Clearly, $M(t)$ is a continuous local martingale with $M(0)=0$. Define $X(t)=X(0)+N(t)+$ $P(t)+M(t)$, where $X(0)=S(0)+E(0)+I(0)+V(0), N(t)=\frac{A}{d-b}\left(1-e^{-(d-b) t}\right)$ and $P(t)=$ $(S(0)+E(0)+I(0)+V(0))\left(1-e^{-(d-b) t}\right)$. By (2.7) we have $S(t)+E(t)+I(t)+V(t) \leq X(t)$ a.s. for all $t \geq 0$, where $N(t)$ and $P(t)$ are continuous adapted increasing processes on $t \geq 0$ with $N(0)=P(0)=0$. From [23, Theorem 3.9], we obtain that $\lim _{t \rightarrow \infty} X(t)<\infty$. Thus, conclusion (2.2) is true. From the fifth equation of system (1.2), it is easy to get that $\limsup _{t \rightarrow \infty} W(t)<$ $\infty$ a.s.

Let

$$
\begin{aligned}
M_{1}(t) & =\int_{0}^{t} S(r) \mathrm{d} B_{1}(r), \widetilde{M}_{1}(t)=\int_{0}^{t} e^{-(d-b)(t-r)} S(r) \mathrm{d} B_{1}(r), \\
M_{2}(t) & =\int_{0}^{t} E(r) \mathrm{d} B_{2}(r), \widetilde{M_{2}}(t)=\int_{0}^{t} e^{-(d-b)(t-r)} E(r) \mathrm{d} B_{2}(r), \\
M_{3}(t) & =\int_{0}^{t} I(r) \mathrm{d} B_{3}(r), \widetilde{M}_{3}(t)=\int_{0}^{t} e^{-(d-b)(t-r)} I(r) \mathrm{d} B_{3}(r), \\
M_{4}(t) & =\int_{0}^{t} V(r) \mathrm{d} B_{4}(r), \widetilde{M}_{4}(t)=\int_{0}^{t} e^{-(d-b)(t-r)} V(r) \mathrm{d} B_{4}(r), \\
M_{5}(t) & =\int_{0}^{t} W(r) \mathrm{d} B_{5}(r), \widetilde{M}_{5}(t)=\int_{0}^{t} e^{-(d-b)(t-r)} W(r) \mathrm{d} B_{5}(r) .
\end{aligned}
$$

Observe the quadratic variations

$$
\begin{gathered}
\left\langle M_{1}(t), M_{1}(t)\right\rangle=\int_{0}^{t} S^{2}(r) \mathrm{d} r \leq\left(\sup _{t \geq 0} S^{2}(t)\right) t \\
\left\langle\widetilde{M_{1}}(t), \widetilde{M_{1}}(t)\right\rangle=\int_{0}^{t} e^{-2(d-b)(t-r)} S^{2}(r) \mathrm{d} r \leq\left(\sup _{t \geq 0} S^{2}(t)\right) t .
\end{gathered}
$$


From the large number theorem for martingales, we have

$$
\lim _{t \rightarrow \infty} \frac{1}{t} M_{1}(t)=0, \lim _{t \rightarrow \infty} \frac{1}{t} \widetilde{M_{1}}(t)=0 \text { a.s. }
$$

Similarly, we also have

$$
\begin{gathered}
\lim _{t \rightarrow \infty} \frac{1}{t} M_{2}(t)=0, \lim _{t \rightarrow \infty} \frac{1}{t} \widetilde{M_{2}}(t)=0, \lim _{t \rightarrow \infty} \frac{1}{t} M_{3}(t)=0, \lim _{t \rightarrow \infty} \frac{1}{t} \widetilde{M_{3}}(t)=0 \text { a.s. } \\
\lim _{t \rightarrow \infty} \frac{1}{t} M_{4}(t)=0, \lim _{t \rightarrow \infty} \frac{1}{t} \widetilde{M_{4}}(t)=0, \lim _{t \rightarrow \infty} \frac{1}{t} M_{5}(t)=0, \lim _{t \rightarrow \infty} \frac{1}{t} \widetilde{M_{5}}(t)=0 \text { a.s. }
\end{gathered}
$$

Observe that

$$
\begin{aligned}
\langle M(t)\rangle= & \frac{\sigma_{1}}{t} \int_{0}^{t} \int_{0}^{u} e^{-(u-r)(d-b)} S(r) \mathrm{d} B_{1}(r) \mathrm{d} u+\frac{\sigma_{2}}{t} \int_{0}^{t} \int_{0}^{u} e^{-(u-r)(d-b)} E(r) \mathrm{d} B_{2}(r) \mathrm{d} u \\
& +\frac{\sigma_{3}}{t} \int_{0}^{t} \int_{0}^{u} e^{-(u-r)(d-b)} I(r) \mathrm{d} B_{3}(r) \mathrm{d} u+\frac{\sigma_{4}}{t} \int_{0}^{t} \int_{0}^{u} e^{-(u-r)(d-b)} V(r) \mathrm{d} B_{4}(r) \mathrm{d} u \\
& +\frac{\sigma_{5}}{t} \int_{0}^{t} \int_{0}^{u} e^{-(u-r)(d-b)} W(r) \mathrm{d} B_{5}(r) \mathrm{d} u \\
= & \frac{\sigma_{1}}{(d-b) t}\left(\int_{0}^{t} S(r) \mathrm{d} B_{1}(r)-\frac{\sigma_{1}}{t} \int_{0}^{t} e^{-(t-r)(d-b)} S(r) \mathrm{d} B_{1}(r)\right) \\
& +\frac{\sigma_{2}}{(d-b) t}\left(\int_{0}^{t} E(r) \mathrm{d} B_{2}(r)-\frac{\sigma_{2}}{t} \int_{0}^{t} e^{-(t-r)(d-b)} E(r) \mathrm{d} B_{2}(r)\right) \\
& +\frac{\sigma_{3}}{(d-b) t}\left(\int_{0}^{t} I(r) \mathrm{d} B_{3}(r)-\frac{\sigma_{3}}{t} \int_{0}^{t} e^{-(t-r)(d-b)} E(r) \mathrm{d} B_{3}(r)\right) \\
& +\frac{\sigma_{4}}{(d-b) t}\left(\int_{0}^{t} V(r) \mathrm{d} B_{4}(r)-\frac{\sigma_{4}}{t} \int_{0}^{t} e^{-(t-r)(d-b)} V(r) \mathrm{d} B_{4}(r)\right) \\
& +\frac{\sigma_{5}}{(d-b) t}\left(\int_{0}^{t} W(r) \mathrm{d} B_{5}(r)-\frac{\sigma_{5}}{t} \int_{0}^{t} e^{-(t-r)(d-b)} W(r) \mathrm{d} B_{5}(r)\right),
\end{aligned}
$$

By using (2.8), (2.9) and (2.10), we get $\lim _{t \rightarrow \infty}\langle M(t)\rangle=0$. An integration from 0 to $t$ and dividing by $t$ on both sides of (2.7), we can get conclusion (2.4). Similarly, from the fifth equation of system (1.2), it is easy to obtain conclusion (2.5). This completes the proof of Lemma 2.2.

\section{EXISTENCE AND UNIQUENESS OF THE GLOBAL POSITIVE SOLUTION}

Theorem 3.1. The solution $(S(t), E(t), I(t), V(t), W(t))$ of system (1.2) is unique for any initial value $(S(0), E(0), I(0), V(0), W(0)) \in \mathbb{R}_{+}^{5}$ on $t \geq 0$, and this solution remains in $\mathbb{R}_{+}^{5}$ with probability one, namely, $(S(t), E(t), I(t), V(t), W(t)) \in \mathbb{R}_{+}^{5}$ for all $t \geq 0$ a.s.

Proof. Since the coefficient of model (1.2) are locally Lipschitz continuous, there exists a unique positive local solution $(S(t), E(t), I(t), V(t), W(t))$ to the model for any initial value

$$
(S(0), E(0), I(0), V(0), W(0)) \in \mathbb{R}_{+}^{5} \text { on } t \in\left[0, \tau_{e}\right),
$$

where $\tau_{e}$ is the explosion time [24]. Next, we prove that the positive solution is global, that is, $\tau_{e}=\infty$ a.s. Let $n_{0} \geq 0$ be sufficiently large, so that $S(0), E(0), I(0), V(0), W(0)$ all lie within the 
interval $\left[1 / n_{0}, n_{0}\right]$. For each integer $n \geq n_{0}$, we define the stopping time as follows

$$
\tau_{n}=\inf \left\{t \in\left[0, \tau_{e}\right): S(t) \notin\left(\frac{1}{n}, n\right), E(t) \notin\left(\frac{1}{n}, n\right), I(t) \notin\left(\frac{1}{n}, n\right), V(t) \notin\left(\frac{1}{n}, n\right) \text { or } W(t) \notin\left(\frac{1}{n}, n\right)\right\},
$$

where $\inf \emptyset:=\infty$ (as usual, $\emptyset$ denotes the empty set). It is easy to see that $\tau_{n}$ is increasing as $n \rightarrow \infty$. Let $\tau_{\infty}=\lim _{t \rightarrow \infty} \tau_{n}$. Hence $\tau_{\infty}<\tau_{e}$ is valid almost surely. So we just need to show that $\tau_{\infty}=\infty$ a.s. If $\tau_{\infty}=\infty$ is false, then there exist two constants $T>0$ and $\varepsilon \in(0,1)$ such that $\mathbb{P}\left\{\tau_{\infty} \leq T\right\}>\varepsilon$. Thus there is an integer $n_{1} \geq n_{0}$ such that

$$
\mathbb{P}\left\{\tau_{n} \leq T\right\} \geq \varepsilon, \quad \text { for any } n>n_{1} .
$$

Define a $C^{2}$-function $\widetilde{V}: \mathbb{R}_{+}^{5} \rightarrow \mathbb{R}_{+}$by

$$
\widetilde{V}(S, E, I, V, W)=S-1-\ln S+E-1-\ln E+I-1-\ln I+V-1-\ln V+W-1-\ln W .
$$

The nonnegativity of this function can be obtained from $u-1-\ln u \geq 0$, for all $u>0$. Applying the generalized Itô's formula to $\widetilde{V}$, we get

$$
\begin{aligned}
\mathrm{d} \widetilde{V}= & \mathscr{L} \widetilde{V} \mathrm{~d} t+\sigma_{1}(S-1) \mathrm{d} B_{1}(t)+\sigma_{2}(E-1) \mathrm{d} B_{2}(t)+\sigma_{3}(I-1) \mathrm{d} B_{3}(t) \\
& +\sigma_{4}(V-1) \mathrm{d} B_{4}(t)+\sigma_{5}(W-1) \mathrm{d} B_{5}(t),
\end{aligned}
$$

where $\mathscr{L} \widetilde{V}: \mathbb{R}_{+}^{5} \rightarrow \mathbb{R}_{+}$is defined

$$
\begin{aligned}
\mathscr{L} \widetilde{V}= & \left(1-\frac{1}{S}\right)\left[A+b(S+V)+\delta V-(d+r) S-\beta S(E+I)-\beta_{1} S W\right] \\
& +\left(1-\frac{1}{E}\right)\left[\beta S(E+I)+\beta_{1} S W-(d+m) E\right]+\left(1-\frac{1}{I}\right)[m E-(d+\alpha) I] \\
& +\left(1-\frac{1}{V}\right)[r S-(\delta+d) V]+\left(1-\frac{1}{W}\right)[k(E+I)-(n \tau+\varepsilon) W] \\
& +\frac{1}{2}\left(\sigma_{1}^{2}+\sigma_{2}^{2}+\sigma_{3}^{2}+\sigma_{4}^{2}+\sigma_{5}^{2}\right) \\
= & +b(S+V)-d S-d E-(d+\alpha) I+k(E+I)-(n \tau+\varepsilon) W-d V \\
& -\frac{A}{S}-\frac{b(S+V)}{S}-\frac{\delta V}{S}+d+r+\beta(E+I)+\beta_{1} W-\frac{\beta S(E+I)}{E}-\frac{\beta_{1} S W}{E} \\
& +d+m-\frac{m E}{I}+d+\alpha-\frac{k(E+I)}{W} \\
& +n \tau+\varepsilon+\delta+d+\frac{1}{2}\left(\sigma_{1}^{2}+\sigma_{2}^{2}+\sigma_{3}^{2}+\sigma_{4}^{2}+\sigma_{5}^{2}\right) \\
\leq & A+4 d+r+m+\alpha+n \tau+\varepsilon+\delta+\frac{1}{2}\left(\sigma_{1}^{2}+\sigma_{2}^{2}+\sigma_{3}^{2}+\sigma_{4}^{2}+\sigma_{5}^{2}\right) \\
& +\left(b \vee(k+\beta) \vee \beta_{1}\right)(S+E+I+V+W)=M_{0}+\lambda(S+E+I+V+W),
\end{aligned}
$$

where $M_{0}=A+4 d+r+m+\alpha+n \tau+\varepsilon+\delta+\frac{1}{2}\left(\sigma_{1}^{2}+\sigma_{2}^{2}+\sigma_{3}^{2}+\sigma_{4}^{2}+\sigma_{5}^{2}\right)$ is a positive constant, and $\lambda=\left(b \vee(k+\beta) \vee \beta_{1}\right)$. By using inequality

$$
S \leq 2(S-1-\ln S)+2 \ln 2 \leq 2 \widetilde{V}(S, E, I, V, W)+2 \ln 2,
$$

we have

$$
S+E+I+V+W \leq 2 \widetilde{V}(S, E, I, V, W)+10 \ln 2 .
$$


It follows that

$$
\begin{aligned}
\mathrm{d} \widetilde{V} \leq & {\left[M_{0}+2 \lambda \widetilde{V}+10 \lambda \ln 2\right] \mathrm{d} t+\sigma_{1}(S-1) \mathrm{d} B_{1}(t)+\sigma_{2}(E-1) \mathrm{d} B_{2}(t) } \\
& +\sigma_{3}(I-1) \mathrm{d} B_{3}(t)+\sigma_{4}(V-1) \mathrm{d} B_{4}(t)+\sigma_{5}(W-1) \mathrm{d} B_{5}(t) .
\end{aligned}
$$

Integrating the both sides of (3.2) from 0 to $\tau_{n} \wedge T$ and taking the expectation, we have

$$
\begin{aligned}
& \left.\mathbb{E} \widetilde{V}\left(S\left(\tau_{n} \wedge T\right)\right), E\left(\tau_{n} \wedge T\right), I\left(\tau_{n} \wedge T\right), V\left(\tau_{n} \wedge T\right), W\left(\tau_{n} \wedge T\right)\right) \\
\leq & \widetilde{V}(S(0), E(0), I(0), V(0), W(0))+2 \lambda \int_{0}^{\tau_{n} \wedge T} \mathbb{E} \widetilde{V}(S, E, I, V, W) \mathrm{d} t+M_{0} T+10 \lambda T \ln 2 .
\end{aligned}
$$

From the Gronwall Lemma, we obtain

$$
\begin{aligned}
& \left.\mathbb{E} \widetilde{V}\left(S\left(\tau_{n} \wedge T\right)\right), E\left(\tau_{n} \wedge T\right), I\left(\tau_{n} \wedge T\right), V\left(\tau_{n} \wedge T\right), W\left(\tau_{n} \wedge T\right)\right) \\
\leq & \left(\widetilde{V}(S(0), E(0), I(0), V(0), W(0))+M_{0} T+10 \lambda T \ln 2\right) e^{2 \lambda\left(\tau_{n} \wedge T\right)} \\
\leq & \left(\widetilde{V}(S(0), E(0), I(0), V(0), W(0))+M_{0} T+10 \lambda T \ln 2\right) e^{2 \lambda T} .
\end{aligned}
$$

Setting $\Omega_{n}=\left\{\tau_{n} \leq T\right\}$, for $n \geq n_{1}$ and using (3.2) we have $\mathbb{P}\left(\Omega_{n}\right) \geq \varepsilon$. Note that, for every $\omega \in \Omega_{n}$, there admits $S\left(\tau_{n}, \omega\right), E\left(\tau_{n}, \omega\right), I\left(\tau_{n}, \omega\right), V\left(\tau_{n}, \omega\right)$, or $W\left(\tau_{n}, \omega\right)$, which equals either $n$ or $1 / n$. Hence

$$
\widetilde{V}\left(S\left(\tau_{n}, \omega\right), E\left(\tau_{n}, \omega\right), I\left(\tau_{n}, \omega\right), V\left(\tau_{n}, \omega\right), W\left(\tau_{n}, \omega\right)\right) \geq\left(\frac{1}{n}-1-\ln \frac{1}{n}\right) \wedge(n-1-\ln n) .
$$

It is then follows from (3.1) and (3.4) that

$$
\begin{aligned}
& \left(\widetilde{V}(S(0), E(0), I(0), V(0), W(0))+M_{0} T+10 \lambda T \ln 2\right) e^{2 \lambda T} \\
\geq & \left.\mathbb{E} \widetilde{V}\left(S\left(\tau_{n} \wedge T\right)\right), E\left(\tau_{n} \wedge T\right), I\left(\tau_{n} \wedge T\right), V\left(\tau_{n} \wedge T\right), W\left(\tau_{n} \wedge T\right)\right) \\
= & \mathbb{E}\left[1_{\Omega_{n}(\omega)} \widetilde{V}\left(S\left(\tau_{n}, \omega\right), E\left(\tau_{n}, \omega\right), I\left(\tau_{n}, \omega\right), V\left(\tau_{n}, \omega\right), W\left(\tau_{n}, \omega\right)\right)\right] \\
\geq & \varepsilon\left[\left(\frac{1}{n}-1-\ln \frac{1}{n}\right) \wedge(n-1-\ln n)\right],
\end{aligned}
$$

where $1_{\Omega_{n}(\omega)}$ is the indicator function of $\Omega_{n}(\omega)$. Letting $n \rightarrow \infty$, we have

$$
\infty>\left(\widetilde{V}(S(0), E(0), I(0), V(0), W(0))+M_{0} T+10 \lambda T \ln 2\right) e^{2 \lambda T}=\infty,
$$

which is a contradiction. Thus, $\tau_{\infty}=\infty$. This completes the proof of Theorem 3.1.

\section{THE EXTINCTION}

Theorem 4.1. Let $(S(t), E(t), I(t), V(t), W(t))$ be the solution of system (1.2) with initial value $(S(0), E(0), I(0), V(0), W(0)) \in \mathbb{R}_{+}^{5}$. If

$$
\mathscr{R}_{0}^{s}=\frac{3(d+m)\left[\beta A(d+m) m+\beta_{1} A m^{2}+k(d-b)(d+m)^{2}\right]}{m(d-b)\left\{(d+m)^{2}\left(d+\alpha+\frac{\sigma_{3}^{2}}{2}\right) \wedge(d+m)^{2}\left(n \tau+\varepsilon+\frac{\sigma_{5}^{2}}{2}\right) \wedge m^{2} \sigma_{2}^{2}\right\}}<1,
$$

then

$$
\lim _{t \rightarrow \infty} E(t)=\lim _{t \rightarrow \infty} I(t)=\lim _{t \rightarrow \infty} W(t)=0 \text { a.s. }
$$

In addition, we have

$$
\lim _{t \rightarrow \infty} \frac{1}{t} \int_{0}^{t} S(r) \mathrm{d} r=\frac{A(d+\delta)}{(d-b)(d+\delta+r)}=S^{0} \text { a.s. }
$$




$$
\lim _{t \rightarrow \infty} \frac{1}{t} \int_{0}^{t} V(r) \mathrm{d} r=\frac{A r}{(d-b)(d+\delta+r)}=V^{0} \text { a.s. }
$$

Proof. Define a $C^{2}$-function $V_{0}: \mathbb{R}_{+}^{5} \rightarrow \mathbb{R}_{+}$by

$$
V_{0}=\ln [m E(t)+(d+m) I(t)+(d+m) W(t)] .
$$

Applying Itô's formula to $V_{0}$ leads to

$$
\begin{aligned}
& \mathrm{d} V_{0}=\left\{\frac{m \beta S(E+I)+m \beta_{1} S W+k(d+m)(E+I)-[(d+m)(d+\alpha) I+(d+m)(n \tau+\varepsilon) W]}{m E+(d+m) I+(d+m) W}\right. \\
& \left.-\frac{m^{2} \sigma_{2}^{2} E^{2}+(d+m)^{2} \sigma_{3}^{2} I^{2}+(d+m)^{2} \sigma_{5}^{2} W^{2}}{2[m E+(d+m) I+(d+m) W]^{2}}\right\} \mathrm{d} t+\frac{m \sigma_{2} E \mathrm{~d} B_{2}(t)}{m E+(d+m) I+(d+m) W} \\
& +\frac{(d+m) \sigma_{3} I \mathrm{~d} B_{3}(t)}{m E+(d+m) I+(d+m) W}+\frac{(d+m) \sigma_{5} W \mathrm{~d} B_{5}(t)}{m E+(d+m) I+(d+m) W} \\
& \leq\left\{\beta S+\frac{m}{d+m} \beta_{1} S-\frac{(d+m)^{2}\left(d+\alpha+\frac{\sigma_{3}^{2}}{2}\right) I^{2}+(d+m)^{2}\left(n \tau+\varepsilon+\frac{\sigma_{5}^{2}}{2}\right) W^{2}+m^{2} \frac{\sigma_{2}^{2}}{2} E^{2}}{[m E+(d+m) I+(d+m) W]^{2}}\right. \\
& \left.+\frac{k(d+m)}{m}\right\} \mathrm{d} t+\frac{m \sigma_{2} E \mathrm{~d} B_{2}(t)}{m E+(d+m) I+(d+m) W}+\frac{(d+m) \sigma_{3} I \mathrm{~d} B_{3}(t)}{m E+(d+m) I+(d+m) W} \\
& +\frac{(d+m) \sigma_{5} W \mathrm{~d} B_{5}(t)}{m E+(d+m) I+(d+m) W} \\
& \leq\left\{-\frac{\left[(d+m)^{2}\left(d+\alpha+\frac{\sigma_{3}^{2}}{2}\right) \wedge(d+m)^{2}\left(n \tau+\varepsilon+\frac{\sigma_{5}^{2}}{2}\right) \wedge m^{2} \frac{\sigma_{2}^{2}}{2}\right]\left(E^{2}+I^{2}+W^{2}\right)}{(d+m)^{2}(E+I+W)^{2}}\right. \\
& \left.+\left(\beta+\frac{m}{d+m} \beta_{1}\right) S+\frac{k(d+m)}{m}\right\} \mathrm{d} t+\frac{m \sigma_{2} E \mathrm{~d} B_{2}(t)}{m E+(d+m) I+(d+m) W} \\
& +\frac{(d+m) \sigma_{3} I \mathrm{~d} B_{3}(t)}{m E+(d+m) I+(d+m) W}+\frac{(d+m) \sigma_{5} W \mathrm{~d} B_{5}(t)}{m E+(d+m) I+(d+m) W} \\
& \leq\left\{-\frac{(d+m)^{2}\left(d+\alpha+\frac{\sigma_{3}^{2}}{2}\right) \wedge(d+m)^{2}\left(n \tau+\varepsilon+\frac{\sigma_{5}^{2}}{2}\right) \wedge m^{2} \frac{\sigma_{2}^{2}}{2}}{3(d+m)^{2}}+\left(\beta+\frac{m}{d+m} \beta_{1}\right) S\right. \\
& \left.+\frac{k(d+m)}{m}\right\} \mathrm{d} t+\frac{m \sigma_{2} E \mathrm{~d} B_{2}(t)}{m E+(d+m) I+(d+m) W}+\frac{(d+m) \sigma_{3} I \mathrm{~d} B_{3}(t)}{m E+(d+m) I+(d+m) W} \\
& +\frac{(d+m) \sigma_{5} W \mathrm{~d} B_{5}(t)}{m E+(d+m) I+(d+m) W} \text {. }
\end{aligned}
$$


Integrating from 0 to $t$ and dividing by $t$ on both sides of (4.1), we get

$$
\begin{aligned}
& \frac{\ln [m E(t)+(d+m) I(t)+(d+m)(t)]}{t} \\
\leq & -\frac{(d+m)^{2}\left(d+\alpha+\frac{\sigma_{3}^{2}}{2}\right) \wedge(d+m)^{2}\left(n \tau+\varepsilon+\frac{\sigma_{5}^{2}}{2}\right) \wedge m^{2} \frac{\sigma_{2}^{2}}{2}}{3(d+m)^{2}} \\
& +\left(\beta+\frac{m}{d+m} \beta_{1}\right) \frac{1}{t} \int_{0}^{t} S(r) d r+\frac{k(d+m)}{m}+\frac{\ln [m E(0)+(d+m) I(0)+(d+m)(0)]}{t} \\
& +\frac{1}{t} M(t)+\frac{1}{t} N(t)+\frac{1}{t} P(t),
\end{aligned}
$$

where

$$
\begin{aligned}
M(t) & =\int_{0}^{t} \frac{m \sigma_{2} E(r) \mathrm{d} B_{2}(r)}{m E(r)+(d+m) I(r)+(d+m) W(r)}, \\
N(t) & =\int_{0}^{t} \frac{(d+m) \sigma_{3} I(r) \mathrm{d} B_{3}(r)}{m E(r)+(d+m) I(r)+(d+m) W(r)}
\end{aligned}
$$

and

$$
P(t)=\int_{0}^{t} \frac{(d+m) \sigma_{5} W(r) \mathrm{d} B_{5}(r)}{m E(r)+(d+m) I(r)+(d+m) W(r)},
$$

which are three local continuous martingales, and $M(0)=N(0)=P(0)=0$. Observe

$$
\begin{gathered}
\langle M(t), M(t)\rangle=\int_{0}^{t}\left(\frac{m \sigma_{2} E(r)}{m E(r)+(d+m) I(r)+(d+m) W(r)}\right)^{2} \mathrm{~d} r \leq \int_{0}^{t} \sigma_{2}^{2} \mathrm{~d} r=\sigma_{2}^{2} t, \\
\langle N(t), N(t)\rangle=\int_{0}^{t}\left(\frac{(d+m) \sigma_{3} I(r)}{m E(r)+(d+m) I(r)+(d+m) W(r)}\right)^{2} \mathrm{~d} r \leq \int_{0}^{t} \sigma_{3}^{2} \mathrm{~d} r=\sigma_{3}^{2} t, \\
\langle P(t), P(t)\rangle=\int_{0}^{t}\left(\frac{(d+m) \sigma_{5} W(r)}{m E(r)+(d+m) I(r)+(d+m) W(r)}\right)^{2} \mathrm{~d} r \leq \int_{0}^{t} \sigma_{5}^{2} \mathrm{~d} r=\sigma_{5}^{2} t, \\
\limsup _{t \rightarrow \infty} \frac{\langle M(t), M(t)\rangle}{t} \leq \sigma_{2}^{2}<\infty, \\
\limsup _{t \rightarrow \infty} \frac{\langle N(t), N(t)\rangle}{t} \leq \sigma_{3}^{2}<\infty,
\end{gathered}
$$

and

$$
\limsup _{t \rightarrow \infty} \frac{\langle P(t), P(t)\rangle}{t} \leq \sigma_{5}^{2}<\infty
$$

By using the large number theorem for martingales, we have

$$
\lim _{t \rightarrow \infty} \frac{1}{t} M(t)=0, \lim _{t \rightarrow \infty} \frac{1}{t} N(t)=0, \lim _{t \rightarrow \infty} \frac{1}{t} P(t)=0 \text { a.s. }
$$


Taking the limit superior of both side of (4.2) and using Lemma 2.2, we obtain

$$
\begin{aligned}
& \limsup _{t \rightarrow \infty} \frac{\ln [m E(t)+(d+m) I(t)+(d+m)(t)]}{t} \\
\leq & -\frac{(d+m)^{2}\left(d+\alpha+\frac{\sigma_{3}^{2}}{2}\right) \wedge(d+m)^{2}\left(n \tau+\varepsilon+\frac{\sigma_{5}^{2}}{2}\right) \wedge m^{2} \frac{\sigma_{2}^{2}}{2}}{3(d+m)^{2}} \\
& +\left(\beta+\frac{m}{d+m} \beta_{1}\right)\left(\limsup _{t \rightarrow \infty} \frac{1}{t} \int_{0}^{t} S(r) d r\right)+\frac{k(d+m)}{m} \\
\leq & -\frac{(d+m)^{2}\left(d+\alpha+\frac{\sigma_{3}^{2}}{2}\right) \wedge(d+m)^{2}\left(n \tau+\varepsilon+\frac{\sigma_{5}^{2}}{2}\right) \wedge m^{2} \frac{\sigma_{2}^{2}}{2}}{3(d+m)^{2}} \\
& +\left(\beta+\frac{m}{d+m} \beta_{1}\right) \frac{A}{d-b}+\frac{k(d+m)}{m}<0 \text { a.s. },
\end{aligned}
$$

which implies that

$$
\lim _{t \rightarrow \infty} E(t)=\lim _{t \rightarrow \infty} I(t)=\lim _{t \rightarrow \infty} W(t)=0 \text { a.s. }
$$

Moreover, using the first equation and the forth equation of system (1.2) yields

$$
\begin{aligned}
& \frac{S(t)-S(0)}{t}+\frac{b+\delta}{\delta+d} \frac{V(t)-V(0)}{t} \\
= & A-\frac{(d-b)(d+\delta+r)}{d+\delta} \frac{1}{t} \int_{0}^{t} S(r) \mathrm{d} r-\frac{1}{t} \int_{0}^{t}\left[\beta S(r)(E(r)+I(r))+\beta_{1} S(r) W(r)\right] \mathrm{d} r \\
& -\frac{\sigma_{1}}{t} \int_{0}^{t} S(r) \mathrm{d} B_{1}(r)-\frac{\sigma_{4}}{t} \int_{0}^{t} V(r) \mathrm{d} B_{4}(r) .
\end{aligned}
$$

Using (4.3) and Lemma 2.2, we conclude

$$
\lim _{t \rightarrow \infty} \frac{1}{t} \int_{0}^{t} S(r) \mathrm{d} r=\frac{A(d+\delta)}{(d-b)(d+\delta+r)}=S^{0} \text { a.s. }
$$

Similarly, integrating from 0 to $t$ and dividing by $t$ on both sides of the forth equation of system (1.2) yield

$$
\frac{V(t)-V(0)}{t}=\frac{r}{t} \int_{0}^{t} S(r) \mathrm{d} r-\frac{\delta+d}{t} \int_{0}^{t} V(r) \mathrm{d} r
$$

which further implies

$$
\lim _{t \rightarrow \infty} \frac{1}{t} \int_{0}^{t} V(r) \mathrm{d} r=\frac{A r}{(d-b)(d+\delta+r)}=V^{0} \text { a.s. }
$$

The proof of Theorem 4.1 is complete.

\section{THE PERSISTENCE IN THE MEAN}

Theorem 5.1. Assume that $\widetilde{\mathscr{R}_{0}^{s}}=\frac{\beta A(d+\delta)}{(d-b)(d+\delta+r)(d+m)}-\frac{\sigma^{2}}{2(d+m)}>1$. Then, for any initial value $(S(0), E(0), I(0), V(0), W(0)) \in \mathbb{R}_{+}^{5}$, the solution $(S(t), E(t), I(t), V(t), W(t))$ of model (1.2) has the following properties

$$
\liminf _{t \rightarrow \infty} \frac{1}{t} \int_{0}^{t} E(r) \mathrm{d} r \geq \frac{(d-b)(d+\delta+r)}{\beta(d+\delta)}\left(\widetilde{\mathscr{R}_{0}^{s}}-1\right)>0 \text { a.s. }
$$




$$
\begin{gathered}
\liminf _{t \rightarrow \infty} \frac{1}{t} \int_{0}^{t} I(r) \mathrm{d} r \geq \frac{m(d-b)(d+\delta+r)}{\beta(d+\delta)(\delta+d)}\left(\widetilde{\mathscr{R}_{0}^{s}}-1\right)>0 \text { a.s. } \\
\liminf _{t \rightarrow \infty} \frac{1}{t} \int_{0}^{t} W(r) \mathrm{d} r \geq \frac{k(\alpha+d+m)(d-b)(d+\delta+r)}{\beta(n \tau+\varepsilon)(d+\delta)(d+\delta)}\left(\widetilde{\mathscr{R}_{0}^{s}}-1\right)>0 \text { a.s. }
\end{gathered}
$$

Proof. Integrating the first, the second and the forth equation of system (1.2) yields

$$
\begin{aligned}
& \frac{S(t)-S(0)}{t}+\frac{E(t)-E(0)}{t}+\frac{b+\delta}{\delta+d} \frac{V(t)-V(0)}{t} \\
= & A-\frac{(d-b)(d+\delta+r)}{d+\delta} \frac{1}{t} \int_{0}^{t} S(r) \mathrm{d} r-(d+m) \frac{1}{t} \int_{0}^{t} E(r) \mathrm{d} r+\frac{1}{t} \int_{0}^{t} \sigma_{1} S(r) \mathrm{d} B_{1}(r) \\
& +\frac{1}{t} \int_{0}^{t} \sigma_{2} E(r) \mathrm{d} B_{2}(r)+\frac{b+\delta}{\delta+d} \frac{1}{t} \int_{0}^{t} \sigma_{4} V(r) \mathrm{d} B_{4}(r) .
\end{aligned}
$$

It is calculated that

$$
\begin{aligned}
\frac{1}{t} \int_{0}^{t} S(r) \mathrm{d} r= & \frac{A(d+\delta)}{(d-b)(d+\delta+r)}-\frac{(d+m)(d+\delta)}{(d-b)(d+\delta+r)} \frac{1}{t} \int_{0}^{t} E(r) \mathrm{d} r \\
& +\frac{(d+\delta)}{(d-b)(d+\delta+r)}\left[\frac{1}{t} \int_{0}^{t} \sigma_{1} S(r) \mathrm{d} B_{1}(r)+\frac{1}{t} \int_{0}^{t} \sigma_{2} E(r) \mathrm{d} B_{2}(r)\right. \\
& \left.+\frac{b+\delta}{\delta+d} \frac{1}{t} \int_{0}^{t} \sigma_{4} V(r) \mathrm{d} B_{4}(r)\right]+\varphi_{1}(t),
\end{aligned}
$$

where

$$
\varphi_{1}(t)=-\frac{d+\delta}{(d-b)(d+\delta+r) t}\left[S(t)-S(0)+E(t)-E(0)+\frac{b+\delta}{\delta+d}(V(t)-V(0))\right] .
$$

Applying Itô's formula, we have

$$
\begin{aligned}
\mathrm{d}(\ln E(t)) & =\left[\frac{\beta S(E+I)}{E}+\frac{\beta_{1} S W}{E}-(d+m)-\frac{1}{2} \sigma_{2}^{2}\right]+\sigma_{2} \mathrm{~d} B_{2}(r) \\
& \geq\left[\beta S(t)-(d+m)-\frac{1}{2} \sigma_{2}^{2}\right]+\sigma_{2} \mathrm{~d} B_{2}(r) .
\end{aligned}
$$

Integrating this inequality from 0 to $t$ and dividing by $t$ on both sides and using equation (5.1), one can obtain that

$$
\begin{aligned}
& \frac{\ln E(t)-\ln E(0)}{t} \\
\geq & \beta \frac{1}{t} \int_{0}^{t} S(r) \mathrm{d} r-(d+m)-\frac{1}{2} \sigma_{2}^{2}+\frac{1}{t} \int_{0}^{t} \sigma_{2} \mathrm{~d} B_{2}(r) \\
= & \frac{\beta A(d+\delta)}{(d-b)(d+\delta+r)}-\frac{\beta(d+m)(d+\delta)}{(d-b)(d+\delta+r)} \frac{1}{t} \int_{0}^{t} E(r) \mathrm{d} r+\frac{\beta(d+\delta)}{(d-b)(d+\delta+r)}[ \\
& \left.\frac{1}{t} \int_{0}^{t} \sigma_{1} S(r) \mathrm{d} B_{1}(r)+\frac{1}{t} \int_{0}^{t} \sigma_{2} E(r) \mathrm{d} B_{2}(r)+\frac{b+\delta}{\delta+d} \frac{1}{t} \int_{0}^{t} \sigma_{4} V(r) \mathrm{d} B_{4}(r)\right] \\
& +\beta \varphi_{1}(t)-(d+m)-\frac{1}{2} \sigma_{2}^{2}+\sigma_{2} \frac{B_{2}(t)}{t} .
\end{aligned}
$$


This inequality can be rewritten as

$$
\begin{aligned}
& \frac{1}{t} \int_{0}^{t} E(r) \mathrm{d} r \\
\geq & \frac{(d-b)(d+\delta+r)}{\beta(d+m)(d+\delta)}\left\{\frac{\beta A(d+\delta)}{(d-b)(d+\delta+r)}+\beta \varphi_{1}(t)-(d+m)-\frac{1}{2} \sigma_{2}^{2}+\sigma_{2} \frac{B_{2}(t)}{t}\right. \\
& +\frac{\beta(d+\delta)}{(d-b)(d+\delta+r)}\left[\frac{1}{t} \int_{0}^{t} \sigma_{1} S(r) \mathrm{d} B_{1}(r)+\frac{1}{t} \int_{0}^{t} \sigma_{2} E(r) \mathrm{d} B_{2}(r)\right. \\
& \left.\left.+\frac{b+\delta}{\delta+d} \frac{1}{t} \int_{0}^{t} \sigma_{4} V(r) \mathrm{d} B_{4}(r)\right]-\frac{\ln E(t)-\ln E(0)}{t}\right\} .
\end{aligned}
$$

An application of the strong law of large number [24] gives

$$
\lim _{t \rightarrow \infty} \frac{B_{2}(t)}{t}=0 \text { a.s. }
$$

By Lemma 2.2, one can obtain that $-\infty<\ln E(t)<\ln \sup _{t \geq 0} E(t)<\infty$ and $\lim _{t \rightarrow \infty} \varphi_{1}(t)=0$ a.s. Taking the inferior limit on both sides of (5.3) yields

$$
\begin{aligned}
& \liminf _{t \rightarrow \infty} \frac{1}{t} \int_{0}^{t} E(r) \mathrm{d} r \\
\geq & \frac{(d-b)(d+\delta+r)}{\beta(d+\delta)}\left[\frac{\beta A(d+\delta)}{(d-b)(d+\delta+r)(d+m)}-\frac{\sigma_{2}^{2}}{2(d+m)}-1\right] \\
= & \frac{(d-b)(d+\delta+r)}{\beta(d+\delta)}\left(\widetilde{\mathscr{R}_{0}^{s}}-1\right) \text { a.s. }
\end{aligned}
$$

Integrating the first, the second, the third and the fifth equation of system (1.2) yields

$$
\begin{aligned}
m & \frac{S(t)-S(0)}{t}+m \frac{E(t)-E(0)}{t}+(d+m) \frac{I(t)-I(0)}{t}+\frac{m(b+\delta)}{\delta+d} \frac{V(t)-V(0)}{t} \\
= & m A-\frac{m(d-b)(d+\delta+r)}{d+\delta} \frac{1}{t} \int_{0}^{t} S(r) \mathrm{d} r \\
& -(d+m)(d+\alpha) \frac{1}{t} \int_{0}^{t} I(r) \mathrm{d} r+m \frac{1}{t} \int_{0}^{t} \sigma_{1} S(r) \mathrm{d} B_{1}(r)+m \frac{1}{t} \int_{0}^{t} \sigma_{2} E(r) \mathrm{d} B_{2}(r) \\
& +(d+m) \frac{1}{t} \int_{0}^{t} \sigma_{3} I(r) \mathrm{d} B_{3}(r)+m \frac{b+\delta}{\delta+d} \frac{1}{t} \int_{0}^{t} \sigma_{4} V(r) \mathrm{d} B_{4}(r) .
\end{aligned}
$$

It is calculated that

$$
\begin{aligned}
\frac{1}{t} \int_{0}^{t} S(r) \mathrm{d} r= & \frac{A(d+\delta)}{(d-b)(d+\delta+r)}-\frac{(d+m)(d+\alpha)(d+\delta)}{m(d-b)(d+\delta+r)} \frac{1}{t} \int_{0}^{t} I(r) \mathrm{d} r \\
& +\frac{(d+\delta)}{m(d-b)(d+\delta+r)}\left[\frac{m}{t} \int_{0}^{t} \sigma_{1} S(r) \mathrm{d} B_{1}(r)+\frac{m}{t} \int_{0}^{t} \sigma_{2} E(r) \mathrm{d} B_{2}(r)\right. \\
& \left.+\frac{d+m}{t} \int_{0}^{t} \sigma_{3} I(r) \mathrm{d} B_{3}(r)+\frac{m(b+\delta)}{\delta+d} \frac{1}{t} \int_{0}^{t} \sigma_{4} V(r) \mathrm{d} B_{4}(r)\right]+\varphi_{2}(t),
\end{aligned}
$$


where

$$
\begin{aligned}
\varphi_{2}(t)= & -\frac{d+\delta}{m(d-b)(d+\delta+r) t}[m(S(t)-S(0))+m(E(t)-E(0))+(d+m)(I(t)-I(0)) \\
& \left.+\frac{m(b+\delta)}{\delta+d}(V(t)-V(0))\right] .
\end{aligned}
$$

Integrating inequality (5.2) from 0 to $t$ and dividing by $t$ on both sides and using equation (5.4) lead to

$$
\begin{aligned}
& \frac{\ln E(t)-\ln E(0)}{t} \\
\geq & \frac{\beta A(d+\delta)}{(d-b)(d+\delta+r)}-\frac{\beta(d+m)(d+\delta)(\delta+d)}{m(d-b)(d+\delta+r)} \frac{1}{t} \int_{0}^{t} I(r) \mathrm{d} r \\
& +\frac{\beta(d+\delta)}{m(d-b)(d+\delta+r)}\left[\frac{m}{t} \int_{0}^{t} \sigma_{1} S(r) \mathrm{d} B_{1}(r)+\frac{m}{t} \int_{0}^{t} \sigma_{2} E(r) \mathrm{d} B_{2}(r)\right. \\
& \left.+\frac{d+m}{t} \int_{0}^{t} \sigma_{3} I(r) \mathrm{d} B_{3}(r)+\frac{m(b+\delta)}{\delta+d} \frac{1}{t} \int_{0}^{t} \sigma_{4} V(r) \mathrm{d} B_{4}(r)\right] \\
& +\beta \varphi_{2}(t)-(d+m)-\frac{1}{2} \sigma_{2}^{2}+\sigma_{2} \frac{B_{2}(t)}{t} .
\end{aligned}
$$

This inequality can be rewritten as

$$
\begin{aligned}
& \frac{1}{t} \int_{0}^{t} I(r) \mathrm{d} r \\
\geq & \frac{m(d-b)(d+\delta+r)}{\beta(d+m)(d+\delta)(\delta+d)}\left\{\frac{\beta A(d+\delta)}{(d-b)(d+\delta+r)}+\beta \varphi_{2}(t)-(d+m)\right. \\
& -\frac{1}{2} \sigma_{2}^{2}+\sigma_{2} \frac{B_{2}(t)}{t}+\frac{\beta(d+\delta)}{m(d-b)(d+\delta+r)}\left[\frac{m}{t} \int_{0}^{t} \sigma_{1} S(r) \mathrm{d} B_{1}(r)\right. \\
& +\frac{m}{t} \int_{0}^{t} \sigma_{2} E(r) \mathrm{d} B_{2}(r)+\frac{d+m}{t} \int_{0}^{t} \sigma_{3} I(r) \mathrm{d} B_{3}(r) \\
& \left.\left.+\frac{m(b+\delta)}{\delta+d} \frac{1}{t} \int_{0}^{t} \sigma_{4} V(r) \mathrm{d} B_{4}(r)\right]-\frac{\ln E(t)-\ln E(0)}{t}\right\} .
\end{aligned}
$$

Taking the limit inferior of both sides (5.5) leads to

$$
\liminf _{t \rightarrow \infty} \frac{1}{t} \int_{0}^{t} I(r) \mathrm{d} r \geq \frac{m(d-b)(d+\delta+r)}{\beta(d+\alpha)(\delta+d)}\left(\widetilde{\mathscr{R}}_{0}^{s}-1\right) \text { a.s. }
$$

On the other hand, integrating the forth equation of system (1.2) from 0 to $t$ and dividing by $t$ on the both sides, we have

$$
\frac{W(t)-W(0)}{t}=\frac{k}{t} \int_{0}^{t}(E(r)+I(r)) \mathrm{d} r-\frac{(n \tau+\varepsilon)}{t} \int_{0}^{t} W(r) \mathrm{d} r+\frac{1}{t} \int_{0}^{t} \sigma_{5} W(r) \mathrm{d} B_{5}(r) .
$$

This equation can be rewritten as

$$
\frac{1}{t} \int_{0}^{t} W(r) \mathrm{d} r=\frac{1}{n \tau+\varepsilon}\left[\frac{k}{t} \int_{0}^{t}(E(r)+I(r)) \mathrm{d} r-\frac{W(t)-W(0)}{t}+\frac{1}{t} \int_{0}^{t} \sigma_{5} W(r) \mathrm{d} B_{5}(r)\right] .
$$


Taking the limit inferior of both side on this equation and using the first and second properties of the Theorem 5.1 and using Lemma 2.2 yields

$$
\liminf _{t \rightarrow \infty} \frac{1}{t} \int_{0}^{t} W(r) \mathrm{d} r \geq \frac{k}{(n \tau+\varepsilon)} \frac{(d-b)(d+\delta+r)(d+\alpha+m)}{\beta(d+\delta)(\alpha+d)}\left(\widetilde{\mathscr{R}_{0}^{s}}-1\right) \text { a.s. }
$$

This completes the proof of Theorem 5.1.

\section{NUMERICAL SIMULATIONS}

In order to confirm the results obtained above, we numerically simulate the path of $S(t)$, $E(t), I(t), V(t)$ and $V(t)$ for stochastic model (1.2) by using Milstein's Higher Order Method [25] with initial value $(S(0), E(0), I(0), V(0), V(0))=(0.8,0.4,0.2,0.67,0.2)$.

In Figure 1 , we choose $A=0.6, d=0.3, m=0.5, \delta=0.2, k=0.1, b=0.2, \varepsilon=0.5, n=3$, $\tau=0.8, \beta=0.0003, \beta_{1}=0.0001, r=0.4, \alpha=0.4, \sigma_{1}=0.18, \sigma_{2}=1.6, \sigma_{3}=0.2, \sigma_{4}=0.18$, $\sigma_{5}=0.18$. Then $\mathscr{R}_{0}^{s}=0.9730<1$ satisfies the condition in Theorem 4.1, that is, the disease dies out with probability one. Moreover, $\lim _{t \rightarrow \infty} \frac{1}{t} \int_{0}^{t} S(r) \mathrm{d} r=3.333, \lim _{t \rightarrow \infty} \frac{1}{t} \int_{0}^{t} V(r) \mathrm{d} r=2.667$ a.s.

In Figure 2, we choose $A=0.3, d=0.3, m=0.5, \delta=0.2, k=0.1, b=0.2, \varepsilon=0.4, n=3$, $\tau=0.8, \beta=0.5, \beta_{1}=0.0001, r=0.4, \alpha=0.4, \sigma_{1}=0.18, \sigma_{2}=0.2, \sigma_{3}=0.2, \sigma_{4}=0.18$, $\sigma_{5}=0.18$. Then $\widetilde{\mathscr{R}}_{0}^{s}=1.0167>1$ satisfies the condition in Theorem 5.1, namely, the disease is persistent in the mean, and in environment, the brucella will persist in long time.

In Figure 3, we choose $\sigma_{5}=3$, and other values are the same as Figure 2. We find when $\sigma_{5}$ increases from 0.18 to 3 , the brucella in environment will dies out.

In Figure 4, we choose $A=0.3, d=0.3, m=0.2, \delta=0.3, k=0.1, b=0.2, \varepsilon=0.4, n=3$, $\tau=0.8, \beta=0.23, \beta_{1}=0.0001, r=0.2, \alpha=0.4$. In figure 4(a) and Figure 4(b), we take $\sigma_{1}=\sigma_{2}=\sigma_{3}=\sigma_{4}=\sigma_{5}=0$, then $\mathscr{R}_{0}>\mathscr{R}_{0}^{\prime}=\frac{\beta A(d+\delta)}{(d-b)(d+\delta+r)(d+m)}=1.035>1$. Hence, the disease is persistent and the brucella in environment will persist in long time; while in Figure $4(\mathrm{c})$ and Figure $4(\mathrm{~d})$, taking $\sigma_{1}=0.08, \sigma_{2}=0.8, \sigma_{3}=0.02, \sigma_{4}=0.18, \sigma_{5}=0.18$, we can find the disease and the brucella in environment will die out under stochastic effects. From the Figure 3 and Figure 4, we obtain that stochastic perturbation is contribute to extinction of the disease.

\section{Discussions}

We investigated a stochastic sheep brucellosis epidemic model with immigration and obtained two thresholds, which control the extinction and the persistence of the disease. Based on model (1.2), the fact there is a unique global positive solution with any positive initial value was established. From Theorems 4.1 and 5.1, the sufficient condition for the extinction and the persistence of the disease were also obtained. 

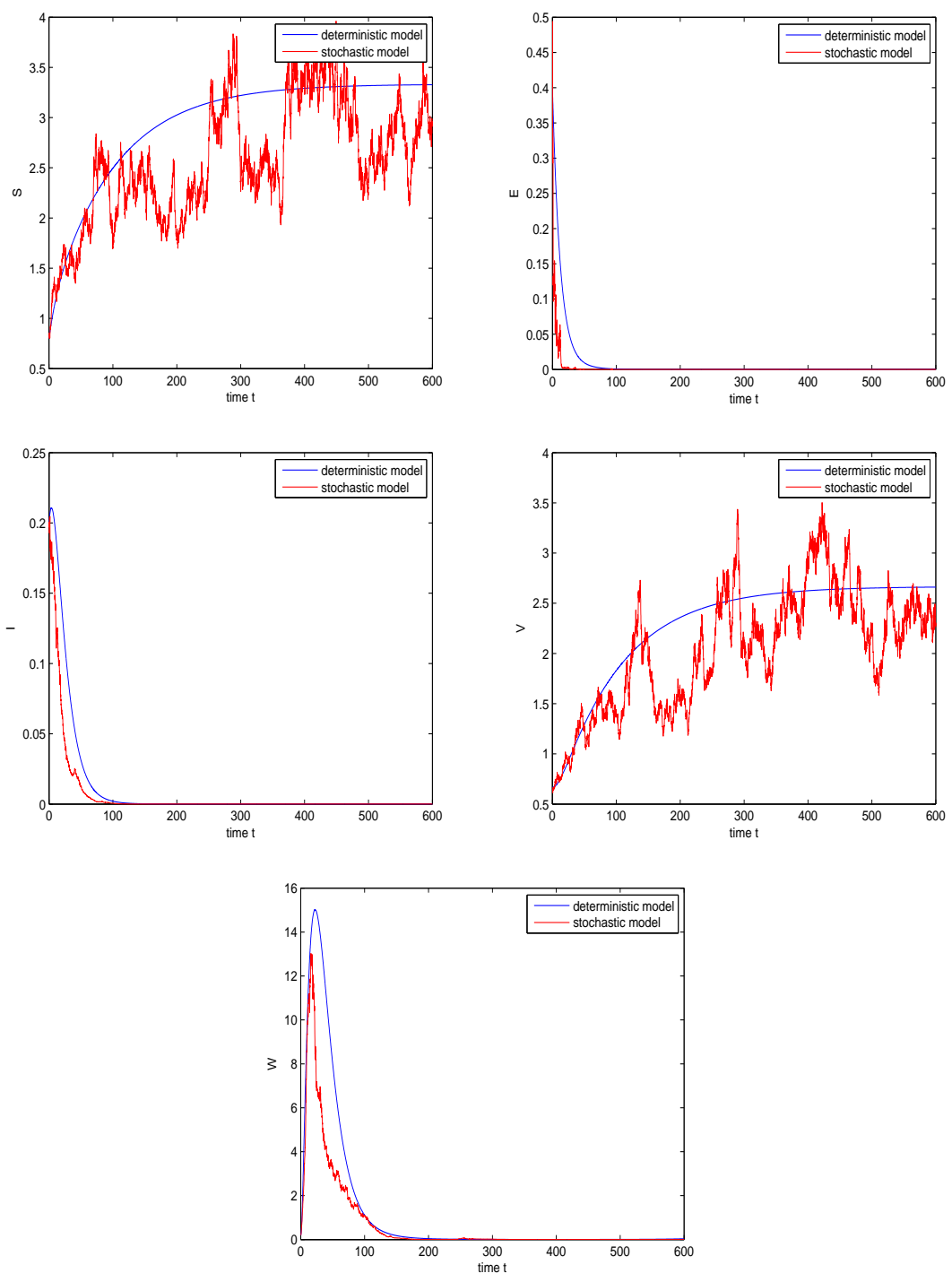

FIGURE 1. When $\mathscr{R}_{0}^{s}<1$, the exposed, infected and the brucella in environment go to extinction
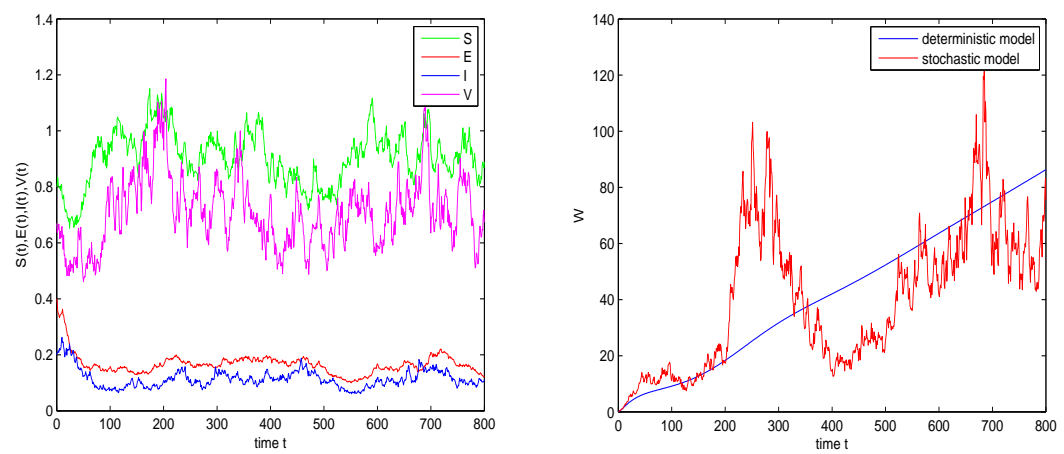

FIGURE 2. When $\widetilde{\mathscr{R}_{0}^{s}}>1$, the disease is persistent and the brucella in environment will persist in long time 


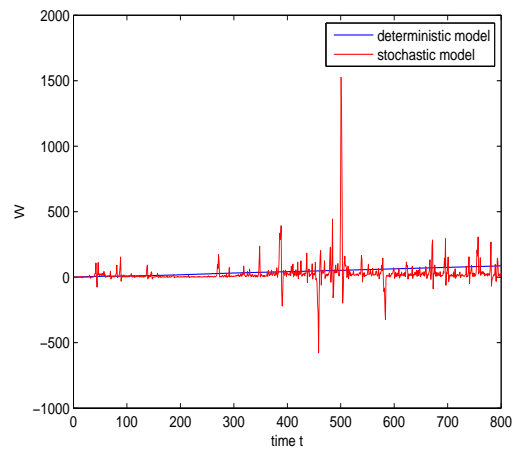

FIGURE 3. When $\sigma_{5}$ increases from 0.18 (the second one of figure 2 ) to 3 , the brucella in environment will die out

(a)

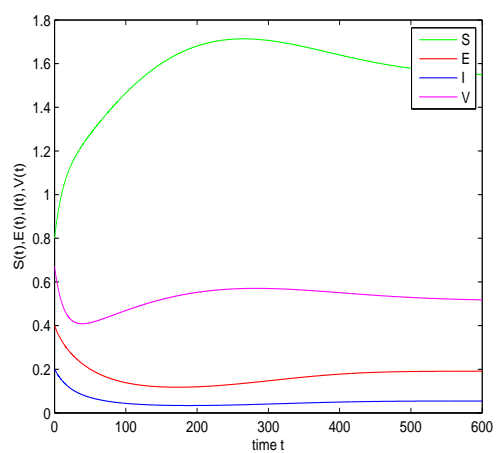

(c)

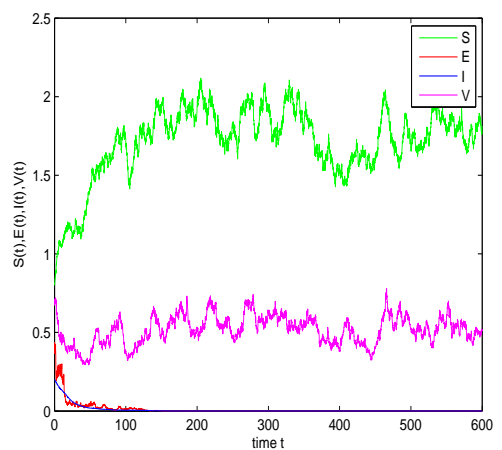

FIGURE 4. A comparison with and without stochastic effect

\section{Acknowledgements}

This work was supported by the National Nature Science Foundation of China, under grant numbers 11861063 and 11771373.

\section{REFERENCES}

[1] M.J. Corbel, Brucellosis: An overview, Emerg. Infect. Dis. 3 (1997), 213-221.

[2] A. MacMillan, Brucellosis: freedom but the risk remains, Cattle Pract. 2 (1994), 469-474.

[3] Y.B. Zhou, X.L. Liu, The research progress in terms of prevalence, incidence reason and control strategies of brucellosis, J. Liaoning Medical Univ. 1 (2010), 81-85. 
[4] F.B. Zhang, Z.W. Li, X.L. La, Multiple-locus variablenumber tander-repeat analysis of Brucella isolates from patients in Xingjiang, China, Int. J. Cliniaal Experimental Med. 8 (2015), 15716-15723.

[5] H.L. Ren, S.Y. Lu, The current research, prevention and control on brucellosis, Chin. Ani. Husband. Vet. Med. 36 (2009), 139-143.

[6] L. Wang, K. Wang, D. Jiang, T. Hayat, Nontrivial periodic solution for a stochastic brucellosis model with application to XinJiang, China, Physica A 510 (2018), 522-537.

[7] G.Q. Sun, Z.K. Zhang, Global stability for a sheep brucellosis model with immigration, Appl. Math. Comput. 246 (2014), 336-345.

[8] B. Alnseba, B. Chahrazed, M. Pierre, A model for ovine brucellosis incorporating direct and indirect transmission, J. Biol. Dyn. 4 (2010), 2-11.

[9] M.T. Li, G.Q. Sun, Y.F. Wu, J. Zhang, Z. Jin, Transmission dynamics of a mutil-group brucellosis model with mixed cross infection in public farm, Appl. Math. Comput. 237 (2014), 582-594.

[10] J. Zinsstag, F. Roth. D. Orkhon, G. Chimed-Ochil, M.Nansalmaa, J. Kolar, P. Vounatsou, A model of animahuman brucellosis transmission in Mongolia, Prev. Vet. Med. 69 (2005), 77-95.

[11] Q. Hou, X.D. Sun, J. Zhang, Y.J. Liu, Y.M. Wang, Z. Jin, Modeling the transmission dynamics of sheep brucellosis in Inner Mongolia Autonomous Region, China, Math. Biosci. 242 (2013), 51-58.

[12] Q. Yang, D. Jiang, N. Shi, C. Ji, The ergodicity and extinction of stochastically perturbed SIR and SEIR epidemic models with saturated incidence, J. Math. Anal. Appl. 388 (2012), 248-271.

[13] A. Gray, D. Greenhalgh, L. Hu, X. Mao, J. Pan, A stochastic differential equation SIS epidemic model, SIAMJ. Appl. Math. 71 (2011), 876-902.

[14] A. Lahrouz, L. Omari, Extinction and stationary distribution of a stochastic SIRS epidemic model with nonlinear incidence, Statist. Probab. Lett. 83 (2013), 960-968.

[15] Y. Zhao, D. Jiang, D. O'Regan, The extinction and persistence of the stochastic SIS epidemic model with vaccination, Physica A 392 (2013), 4916-4927.

[16] Y. Zhao, D. Jiang, Dynamics of stochastically perturbed SIS model with vaccination, Abstr. Appl. Anal. 2013 (2013), 1-12.

[17] C. Ji, D. Jiang, Threshold behaviour of a stochastic SIR model, Appl. Math. Model. 38 (2014), 5067-5079.

[18] Y. Zhao, D. Jiang, The threshold of a stochastic SIRS epidemic model with saturated incidence, Appl. Math. Lett. 34 (2014), 90-93.

[19] Q. Liu, D. Jiang, N. Shi, Threshold behavior in a stochastic SIQR epidemic model with standard incidence and regime switching, Appl. Math. Comput. 316 (2018), 310-325.

[20] F. Li, X. Meng, X. Wang, Analysis and numerical simulation of a stochaListic SEIQR epidemic system with quarantine-adjusted incidence and imperfect vaccination, Comput. Math. Methods Med. 2018 (2018), Article ID 7873902.

[21] Y. Chen, B.Y. Wen, Z.D. Teng, The global dynamics for a stochastic SIS epidemic model with isolation, Physica A 492 (2018), 1604-1624.

[22] N. Ikeda, S. Watanabe, A comparision theorem for solutions of stochastic differential equations and its applications, Osaka J. Math. 14 (1997), 619-633.

[23] X. Mao, Stochastic Differential Equations and Applications, 2nd ed, Horwood, Chichester, 2008.

[24] X. Mao, Stochastic Differential Equations and Applications, Horwood, Chichester, 1997.

[25] D.J. Higham, An algorithmic introduction to numerical simulation of stochastic differential equations, SIAM Rev. 43 (2001), 525-546. 